\title{
Shape memory alloy honeycombs: experiments \& simulation
}

\author{
John A. Shaw*† Chris Churchill $\$$ \\ Nicolas Triantafyllidis ${ }^{\dagger}$ Petros Michailidis ${ }^{\S}$ \\ Aerospace Engineering \\ The University of Michigan
}

\author{
David Grummon \\ John Foltz
}

Metallic foams and honeycombs, with their light-weight, high specific stiffness, and well-developed energy absorption characteristics, are of obvious utility in engineering applications. However, these structures, often made of aluminum, suffer permanent deformation after crushing. Cellular structures made from shape-memory alloys (SMAs) are particularly intriguing for their potential to deliver shape memory and/or superelasticity in a light-weight material. Realization of open-celled Nitinol has recently become possible via a (newly discovered by Profs. D. Grummon at Michigan State Univ. and J. Shaw at Univ. of Michigan) transient-liquid reactive brazing system for creating robust metallurgical Nitinol-Nitinol bonds. With this technique, prototype sparse cellular honeycomb structures have been made and tested, showing up to $50 \%$ repeatedly recoverable strains.

Two different microgeometry NiTi honeycombs have been tested, a hexagonal for isothermal conditions and a corrugated for thermal cycling. Moreover, the isothermal experiments are simulated using a model that accounts for finite rotations and hysteretic responce of the ligaments and geometric imperfections of the finite size samples.

\section{Nomenclature}

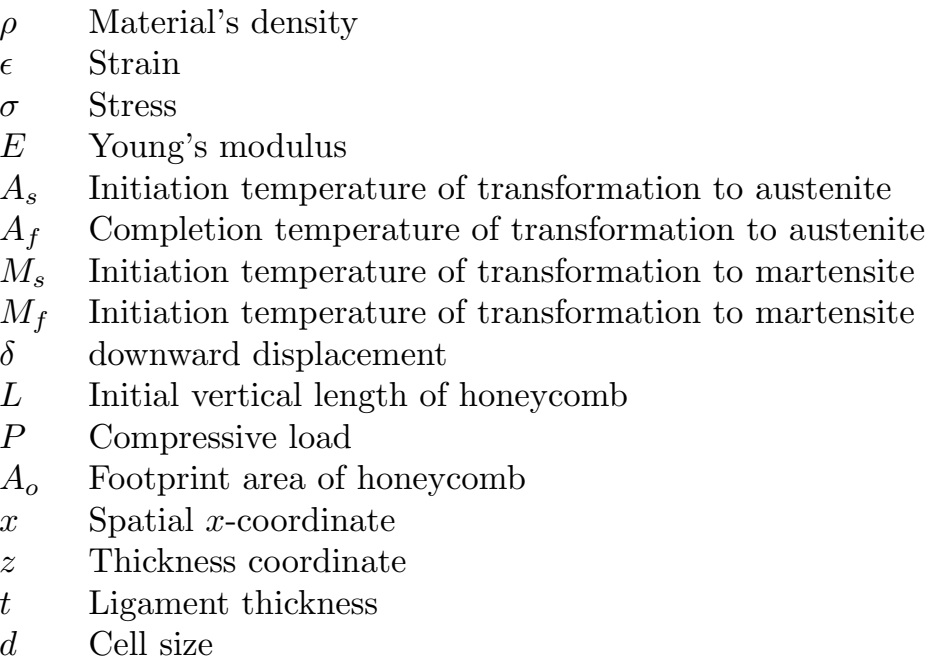

\footnotetext{
*Corresponding author, jashaw@umich.edu

${ }^{\dagger}$ Professor,Aerospace Engineering Department,The University of Michigan, Ann Arbor, MI 48109-2140

$\ddagger$ Professor, Chemical Engineering and Materials Science Department, Michigan State University, East Lansing, MI 488241226

$\S$ Graduate Student, Aerospace Engineering Department, University of Michigan, Ann Arbor, MI 48109-2140

ฯ Graduate Student, Chemical Engineering and Materials Science Department, Michigan State University, East Lansing, MI $48824-1226$
} 
$e \quad$ Axial strain of beam's centroidal axis

$\lambda \quad$ Stretch ratio of beam's centroidal axis

$\kappa \quad$ Curvature of beam's centroidal axis

$v(x) \quad x$-displacement of point on centroidal axis, initially at $x$

$w(x) z$-displacement of point on centroidal axis, initially at $x$

$N \quad$ Axial force

$M \quad$ Bending moment

$\xi \quad$ Internal variable of the material model

$\beta \quad$ Material parameter

$\sigma_{u} \quad$ Upper yield surface stress

$\sigma_{l} \quad$ Lower yield surface stress

$E_{t} \quad$ Modulus during phase transformation

$\epsilon_{1 u} \quad$ Nucleation strain during loading

$\epsilon_{2 l} \quad$ Nucleation strain during unloading

$\dot{f} \quad$ Rate of change of parameter $f$

Subscript

$s \quad$ Property of solid material

Superscript

* $\quad$ Effective property of honeycomb

\section{Introduction}

Metallic foams and honeycombs, with their light-weight, high specific stiffness, and well-developed energy absorption characteristics, are of obvious utility in engineering applications. ${ }^{1,2}$ However, these structures, often made of aluminum, suffer permanent deformation after crushing. Cellular structures made from shapememory alloys (SMAs) are particularly intriguing for their potential to deliver shape memory and/or superelasticity in a light-weight material. While porous forms of NiTi have been made near $50 \% \mathrm{dense}^{3}$ using powder metallurgy techniques, the difficulty of joining Nitinol to itself, while preserving robust adaptive properties, has hampered the realization of built-up, very low-density, cellular honeycombs from NiTi-based SMAs.

The maximum tensile strain recovery obtainable from a monolithic Nitinol polycrystal is in the range of 5 to $8 \%$, in the low-cycle limit, and is less than $2.5 \%$ when high cycle fatigue is a factor. These limits can, however, be substantially exceeded by exploiting bending of thin ligaments during in-plane loading of open-cell structures. During deformation, open structures also cope more effectively with latent heat effects associated with the underlying displacive transformations in SMAs. Thermal inertia tends to dominate the response time of SMA actuators, and furthermore, can cause hypersensitive rate dependencies. ${ }^{4-6}$ The thermal time constant scales with the structure's volume-to-surface area ratio, which is potentially greatly reduced with sparse cellular materials. Furthermore, one reason that Nitinol is most commonly available in wire or thin strip form is that larger sections have poorer properties due to inhomogeneous quenching rates after annealing. By contrast, open cell structures, even those with substantial mass, can be effectively quenched during heat treatment to develop optimal properties, since the surface area/volume ratio scales more favorably.

Effective properties can be achieved with SMA honeycombs that are not possible with conventional aluminum honeycombs as described in Table 1. Consider a honeycomb structure with the same cell size and topology made from high strength aluminum with a modulus $E=70 \mathrm{GPa}$ (the same modulus as austenitic Nitinol) and yield strength of $350 \mathrm{GPa}$. Yielding can be avoided by designing the honeycomb such that the maximum strain does not exceed $\approx 0.5 \%$ during crushing. Large-displacement lateral compression of a honeycomb (with ligament thickness, $t$, and cell size, $d$ ) relies primarily on bending of the ligaments as cell rows are flattened. ${ }^{7}$ The local extreme fiber strain is of order $t / d$, since $d$ is a measure of the radius of curvature of the initial cell. Because aluminum must remain within $0.5 \%$ strain to avoid yielding, but the SMA can be strained to about 5\%, the ligament thickness in the aluminum honeycomb must be thinner than the corresponding SMA thickness by a factor of 10. The initial effective elastic modulus of the honeycomb scales with $(t / d)^{3}$, so the thinner ligament in the aluminum honeycomb reduces its effective modulus by a factor of 1000 compared to the SMA honeycomb. In addition, the effective crushing stress scales as $(t / d)^{2}$, 
so the effective crushing stress in the aluminum honeycomb would be roughly $1 / 100$ to that of the Nitinol honeycomb with the same macroscopic dimensions and cell topology.

Table 1. Expected scaling laws and comparison between conventional aluminum honeycomb and Nitinol honeycomb for the same recovery strain. Subscript ' $s$ ' denotes properties of the solid material, and superscript $*$ denotes effective properties of the honeycomb. Numerical values shown are order of magnitude estimates.

\begin{tabular}{|l|c|c|c|}
\hline Property & Scaling & Aluminum honeycomb & NiTi honeycomb \\
\hline relative density & $\frac{\rho^{*}}{\rho_{\mathrm{s}}} \sim \frac{t}{d}$ & $10^{-3}$ & $10^{-2}$ \\
max bending strain & $\varepsilon_{\mathrm{s}, \max } \sim \frac{t}{d}$ & $10^{-3}$ & $10^{-2}$ \\
effective plateau stress & $\frac{\left|\sigma^{*}\right|}{\sigma_{\mathrm{s}}} \sim\left(\frac{t}{d}\right)^{2}$ & $10^{-6}$ & $10^{-4}$ \\
effective modulus & $\frac{E^{*}}{E_{\mathrm{s}}} \sim\left(\frac{t}{d}\right)^{3}$ & $10^{-9}$ & $10^{-6}$ \\
\hline
\end{tabular}

Besides the individual advantages of these two classes of materials, the combination can provide adaptive properties beyond those of a conventional monolithic SMA in terms of amplified strain recovery and thermal response time. Both attributes, isothermal recovery (superelasticity) and thermal shape recovery, are of obvious utility in the context of active structures. Furthermore, if Nitinol honeycombs could be realized as open-cell, layered structures by simply building them up from wrought SMA sheet, strip, tube, or wire (pre-engineered and acquired off-the-shelf), such materials might become economically attractive. Our goal, therefore, is to design and fabricate shape-memory and superelastic metal honeycombs that combine amplified shape recovery with high specific stiffness, excellent fatigue-resistance, low density, good thermal and chemical stability, and even biocompatibility. SMA honeycombs could prove useful in defense and aerospace applications as thermally-active multifunctional materials, highly resilient structures, lightweight armor, or in novel vibration damping systems.

\section{A New Fabrication Method for Cellular Nitinol Structures}

Honeycomb structures built up from wrought SMAs are a viable alternative to foamed or porous metals if a method can be found to join individual corrugated or dimpled sheets or strips. The joining method used to create an open topology must not only provide a robust metallurgical bond, but must also be derivable from a simple, clean, and cost-effective batch process. While a few specialized techniques for soldering and welding Nitinol have been developed over the years, until now no low-cost joining method capable of producing tough metallurgical bonds in complex multiple-contact structures, such as honeycombs or spaceframes, has been available.

We have recently discovered a solution in the form of a niobium-based braze that has promising metallurgical characteristics. When niobium is brought into contact with conventional wrought Nitinol at elevated temperature, the existence of a quasibinary eutectic minimum in the liquidus temperature drives local melt-

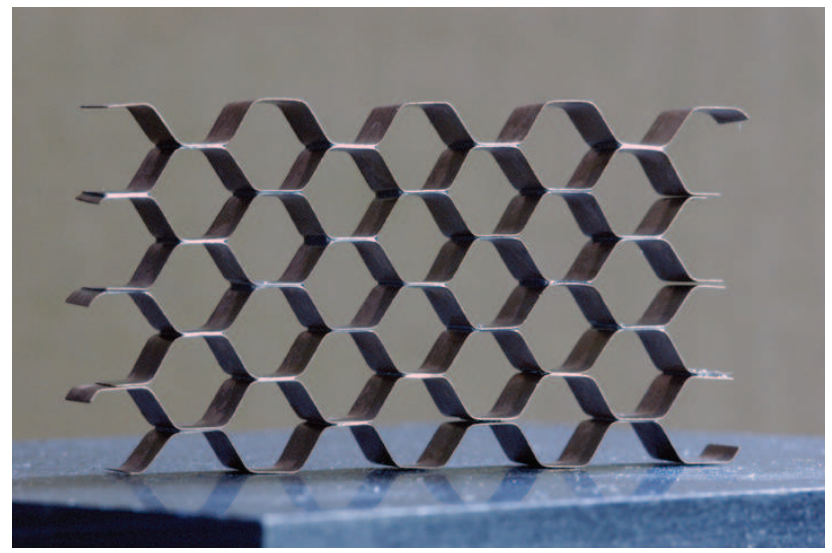

Figure 1. A superelastic Nitinol honeycomb structure with relative density of $<5 \%$, fabricated by joining individual corrugated Nitinol strips using the newly developed brazing system. The approximate ligament thickness is $t=0.1$ mm (except at the double layered joints), and the approximate cell diameter is $d=5.4 \mathrm{~mm}$. 
ing. The liquid produced has a composition near the quasibinary $\mathrm{TiNi}-\mathrm{Nb}$ eutectic at $\mathrm{Ni}_{38} \mathrm{Ti}_{36} \mathrm{Nb}_{26}$. It aggressively wets both pure niobium and $\mathrm{NiTi}$, and is therefore self-fluxing in the sense that it can dissolve oxide scales. Both solid phases that appear on eutectic freezing (B2-NiTi and bcc-Nb) are ductile, leading to braze joints that are strong, tough, corrosion resistant, and thermally stable. None of the alloy constituents is exotic or unreasonably expensive, and the end product is probably biocompatible.

Honeycomb specimens, such as the one shown in Fig. 1, were fabricated at Michigan State University from $5.2 \mathrm{~mm}$ wide rolled, superelastic, nickel-rich Nitinol strip obtained from Memry Corporation, Brookfield CT. Strip thickness $0.1 \mathrm{~mm}$ was used for the hexagonal microgeometry specimen and $0.2 \mathrm{~mm}$ for the corrugated one. Based on differential scanning calorimetry the strip material had the following transformation temperatures: $A_{s}=25{ }^{\circ} \mathrm{C}, A_{f}=7{ }^{\circ} \mathrm{C}, M_{s}=-54{ }^{\circ} \mathrm{C}$, and $M_{f}=-75{ }^{\circ} \mathrm{C}$. The strips were first shape-set into corrugated form at $500{ }^{\circ} \mathrm{C}$ for 10 to 30 minutes using stainless steel dies. After shape-setting, the corrugated strips were etched in a solution containing $50 \mathrm{~mL} \mathrm{HNO}, 38 \mathrm{~mL} \mathrm{HCl}$, and $17 \mathrm{~mL} \mathrm{HF}$ to remove surface oxides. The honeycomb layup was assembled with small 50.8 micron thick square pieces ( $3 \mathrm{~mm} \mathrm{x}$ $3 \mathrm{~mm}$ ) of $99.7 \%$ pure niobium foil placed between the contacting faces.

The assembled layup was lightly secured in TZ-molybdenum support fixtures with no fluxing agent and washed in acetone and ethanol. Brazing was conducted in a Centorr M60 tungsten-element diffusion-pumped vacuum furnace evacuated to a base pressure of $7 \times 10^{-6}$ Torr and then heated quickly to between 1175 and $1200{ }^{\circ} \mathrm{C}$, held for a brief period, and then furnace cooled. After removal from the vacuum furnace, the as-brazed honeycombs were given an aging treatment near $500{ }^{\circ} \mathrm{C}$ prior to compression testing.

\section{Experiments}

Experiments were performed on prototype Nitinol honeycombs that exhibit an amplified form of superelasticity and shape memory recovery. An example of each is discussed below.

\section{III.A. Isothermal Superelastic Experiment}

An in-plane crushing experiment was performed at the University of Michigan using an Instron 5585 electromechanical testing machine in room temperature air $\left(22{ }^{\circ} \mathrm{C}\right)$ under quasistatic displacement control at an effective strain rate of $\dot{\delta} / L=3.33 \times 10^{-3} \mathrm{~s}^{-1}$. Digital optical photographs were taken periodically using a $6 \mathrm{M}$ pixel Nikon D100 camera fitted with a $105 \mathrm{~mm}$ Micro Nikkor lens.

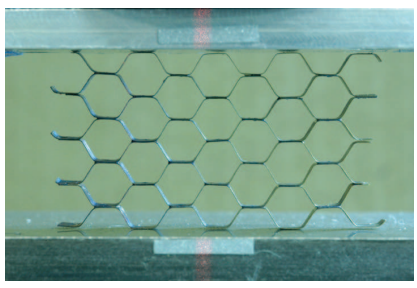

(a)

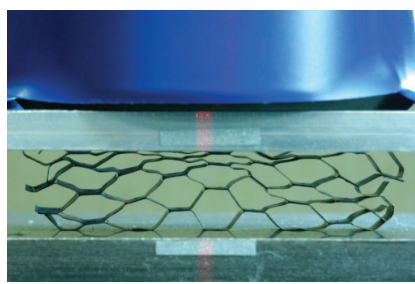

(b)

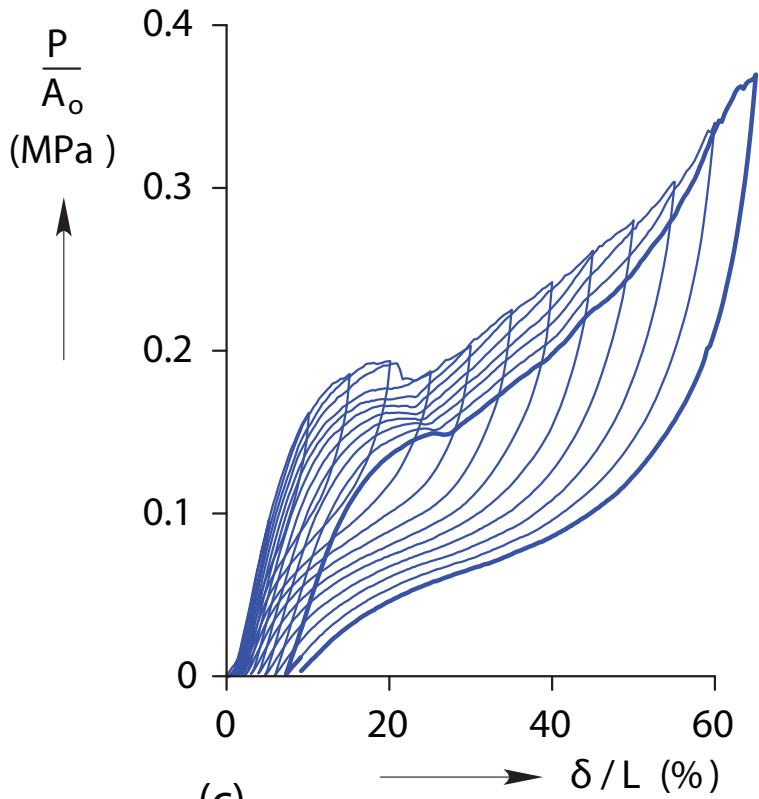

(c)

Figure 2. Photograph (a) of undeformed specimen and (b) of deformed specimen at strain $\delta / \mathrm{L}=52 \%$. In (c) is the stress-strain diagram for the isothermal mechanical responce. 
Figure 2a shows an image of a Nitinol honeycomb specimen (4-1/2 cells high by about 7 cells wide) at the start of the experiment, and Fig. 2b shows a deformed configuration. In the same figures we also show the initial vertical length $(L)$ between compression platens and the downward displacement $(\delta)$ of the upper platen. The silver strips seen on the front of the platens are reflective tags used with an EIR model LE-05 laser extensometer to accurately measure platen displacement and to serve as a cross-check on the strain measurement derived from the crosshead displacement. The mechanical response shown in Fig. 2c is reported as the compressive load $(P)$ divided by the projected cross-sectional area, $A_{o}$, (the total footprint area, including the area of the incomplete cells at the edges) perpendicular to the loading direction, to give a macroscopic measure of compressive stress. An incremental strain history with load-unload cycles in $5 \%$ steps were imposed to probe the effective superelastic strain recovery properties and residual strain. A limit load is seen in the response on the fifth cycle, near $22 \%$ strain, when a local band of highly deformed cells formed, yet the honeycomb recovers to a nearly uniform configuration upon unloading. The final load-unload cycle, taken to $65 \%$ compressive strain, is shown by the bold line in the figure. Although the closure of the loop is not perfect (leaving about 1.3\% relative unrecovered strain), it clearly shows a wide hysteresis and nonlinear behavior typical of a superelastic material undergoing bending deformation. The absolute residual strain was $<10 \%$ demonstrating a strain recovery of over $55 \%$. Subsequent cycles (not shown) show shakedown to a relatively stable hysteresis loop. Other similar superelastic experiments on corrugated Nitinol structures are reported in 8.

\section{III.B. Shape Memory Experiment}

A second experiment was performed on a wavy-corrugated honeycomb specimen as shown in Fig. 3a to demonstrate shape memory behavior. The ligament thickness in this case was $t=0.2 \mathrm{~mm}$ (twice as thick as before), yet the initial radius curvature, $1 / d$, was similar to the average $1 / d$ of the previous hexagonal honeycomb. Figure 3b shows the stress-strain-temperature measurements conducted in an oil-chilled bath. First, the specimen was heated to $40{ }^{\circ} \mathrm{C}$ to ensure the specimen started in the austenite phase, and then it was then cooled (magenta line) nearly stress-free to about $-20{ }^{\circ} \mathrm{C}$, resulting in only a small change of about $2 \%$ compressive strain. We suspect this small strain was due to either the crystallographic texture in the original strip material or some small residual stresses that resulting from the fabrication process. The temperature was then held at $-20^{\circ} \mathrm{C}$, and a load-unload displacement cycle was performed (blue line). The maximum compressive strain was about $37 \%$ during loading, and the residual strain upon unloading

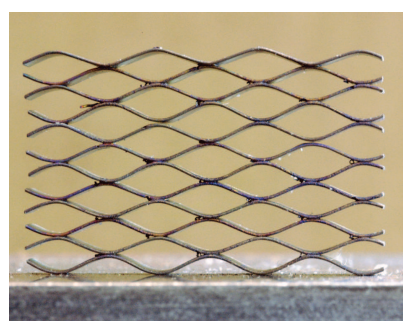

(a)

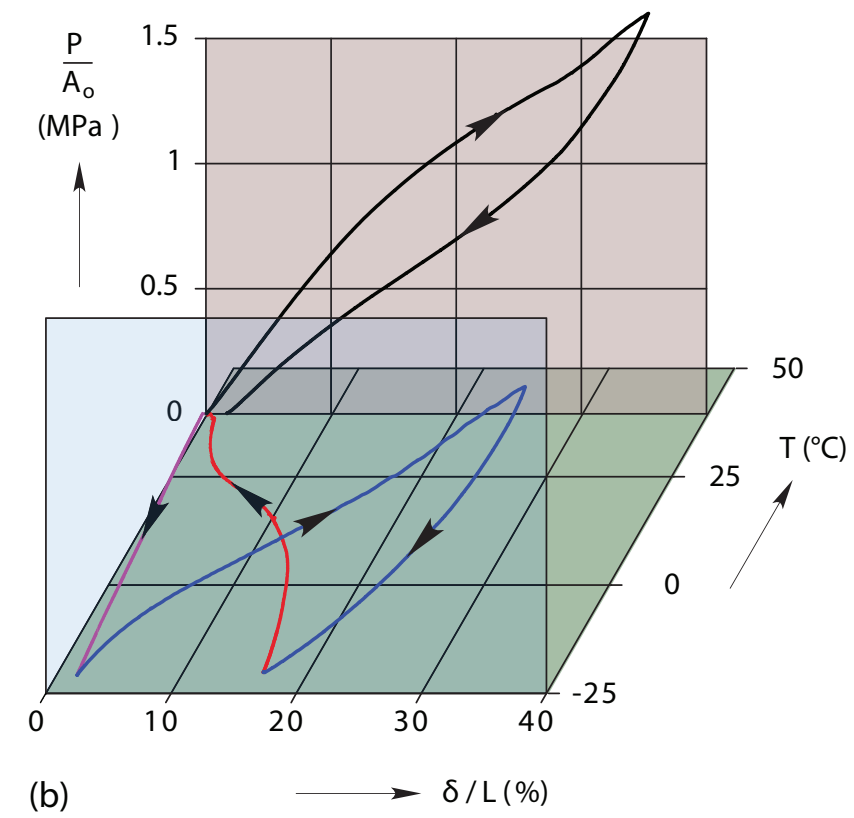

Figure 3. Results of shape memory experiment on a corrugated Nitinol honeycomb: (a) initial photograph of the specimen before the experiment, (b) thermomechanical response showing the shape memory effect with stress-free cooling (magenta line), isothermal load-unload (blue), then stress-free heating (magenta). A subsequent superelastic response is also shown (black line) for reference. 
was about $17 \%$, showing significant spring back even during the martensite response. Next, the load was removed and the bath was heated to $40{ }^{\circ} \mathrm{C}$, and the strain was recovered to within $1 \%$ of the original configuration. This demonstrates a significantly larger strain recovery than can be obtained with monolithic Nitinol. Although not done here, we expect to recover even larger strains by loading further during the martensitic (cold) loading segment. A superelastic load-unload cycle was then performed at $40{ }^{\circ} \mathrm{C}$. Note that the effective stress levels are accordingly higher than the results of the previous hexagonal honeycomb due to the two-fold larger $t / d$ ratio and the different cell topology.

\section{Modelling \& Simulation of Isothermal Experiment}

Simulations have been done for the case of isothermal experiments on the 4-1/2 x 7 cell with hexagonal geometry shown in Fig. 2a, using small-strain, finite displacement and rotation nonlinear beam kinematics for the ligaments. Although a detailed presentation of the corresponding theory is given in Ref. 9, a brief description of the model is given here for completeness.

Adopting the classic Bernulli-Navier-Euler hypothesis for the kinematics of the beam (planarity of deformed sections perpendicular to centroidal axis and their normality to the deformed centroidal axis) the axial strain $\epsilon(x, z)$ at a point with initial coordinates $(x, z)$ in the local frame of each beam is taken to be (see Fig. 4)

$$
\epsilon=e+z \kappa
$$

Here e is the axial strain of the beam's centroidal axis

$$
e=\lambda-1 \quad \lambda=\left[\left(1+v_{, x}\right)^{2}+\left(w_{, x}\right)^{2}\right]^{1 / 2}
$$

where $\lambda$ is the stretch ratio of the beam's centroidal axis expressed in terms of the $x$ and $z$ displacements $v(x)$ and $w(x)$ of a point on this axis initially at $x$. The bending strain $\kappa$ is given in terms of the same displacements by

$$
\kappa=\left[\left(1+v_{, x}\right) w_{, x x}-w_{, x} v_{, x x}\right] / \lambda^{2}
$$

The work-conjugate quantities associated to the axial $(e)$ and bending $(\kappa)$ strains are the axial force $(N)$ and bending moment $(M)$ given by

$$
N=\int_{-\frac{t}{2}}^{\frac{t}{2}} \sigma \mathrm{d} z \quad M=\int_{-\frac{t}{2}}^{\frac{t}{2}} \sigma z \mathrm{~d} z
$$

where $t$ is the beam thickness.

The variational form use to obtain the displacement field for each ligament is (in view of absence of distributed external loads)

$$
\int_{0}^{L}(N \delta e+M \delta \kappa) \mathrm{d} x=0
$$

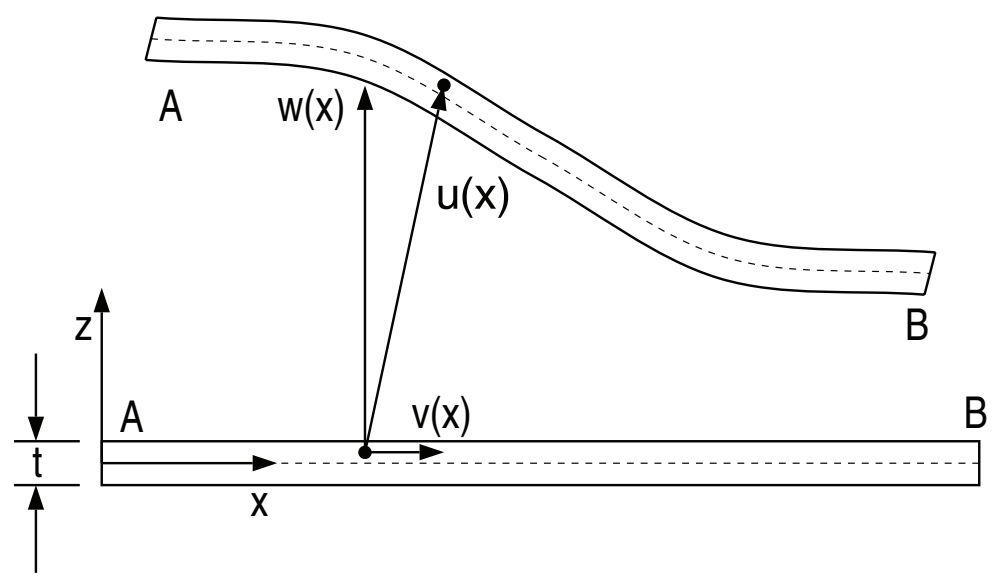

Figure 4. Undeformed and deformed configuration for beam element of thickness $t$. 
It should be pointed out here that the particular choice, made in Eq. (2) and (3) for the kinematics of the beam leads to a consistent theory, i.e. the Euler-Lagrange equations derived from Eq. (5) coincide with the exact equilibrium equations of the beam in the current configuration. These latter set of equations can be obtained directly from equilibrium considerations of a beam segment with no distributed load.

The last element required to complete the description of the behavior of the cell walls is the constitutive law for the cell wall material. To this end the constitutive equation for the tensile region $\sigma>0$ for the stress $(\sigma(x, z))$ - strain $(\epsilon(x, z))$ response at a material point with reference coordinates $(x, z)$ in each ligament is given by

$$
\sigma=E(e-\beta \xi)
$$

where $0 \leq \xi \leq 1$ is the hysteretic model's internal variable and $\beta$ a material parameter. The material's incremental response is given by the rate form Eq. (6) where in the elastic range

$$
\sigma_{u}(\xi)<\sigma<\sigma_{l}(\xi) \quad \dot{\xi}=0
$$

while for stress states on the upper $\sigma_{u}(\xi)$ or lower $\sigma_{l}(\xi)$ yield surfaces

$$
\begin{array}{ll}
\sigma_{u}(\xi)=\sigma & \dot{\xi}=\left\{\begin{array}{lll}
\frac{E-E_{t}}{\beta} \dot{\epsilon} & \dot{\epsilon}>0 & \text { loading } \\
0 & \dot{\epsilon}<0 & \text { unloading }
\end{array}\right. \\
\sigma_{l}(\xi)=\sigma & \dot{\xi}=\left\{\begin{array}{lll}
\frac{E-E_{t}}{\beta} \dot{\epsilon} & \dot{\epsilon}<0 & \text { loading } \\
0 & \dot{\epsilon}>0 & \text { unloading }
\end{array}\right.
\end{array}
$$

A schematic representation of the various parameters of this hysteretic model is given in Fig. 5. By prescribing $E, E_{t}, \beta, \epsilon_{1 u}$ and $\epsilon_{2 l}$ the tensile behavior of the model is fully determined. An analogous construction is also adopted for the compressive region $\sigma>0$ of the SMA's response. Notice that there is no need to have tension/compression symmetry for the material response of the cell walls, a feature adopted in our numerical simulations to better match the calculations with experimental results.

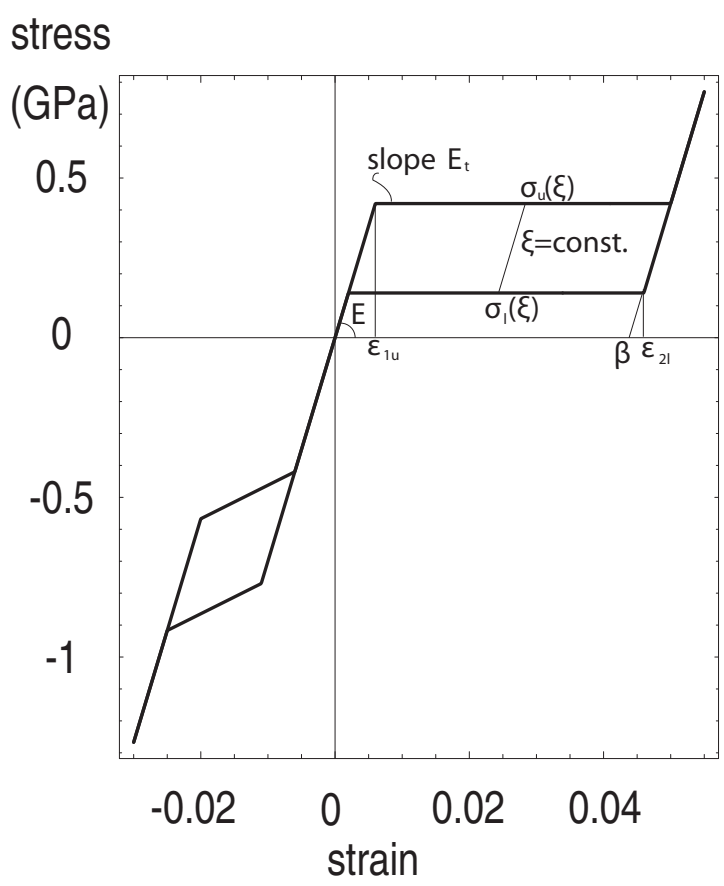

(a)
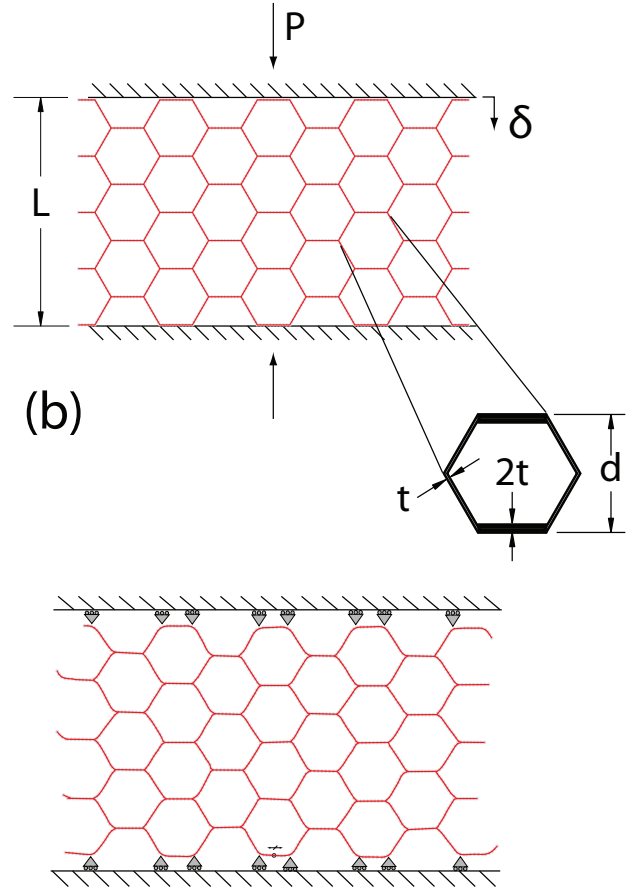

(c)

Figure 5. Modelling of isothermal experiment: (a) Hysteretic stress - strain law used in numerical calculations, (b) Idealized perfect geometry with definition of parameters, (c) Imperfect geometry (created from a digitized picture of the actual specimen) . 

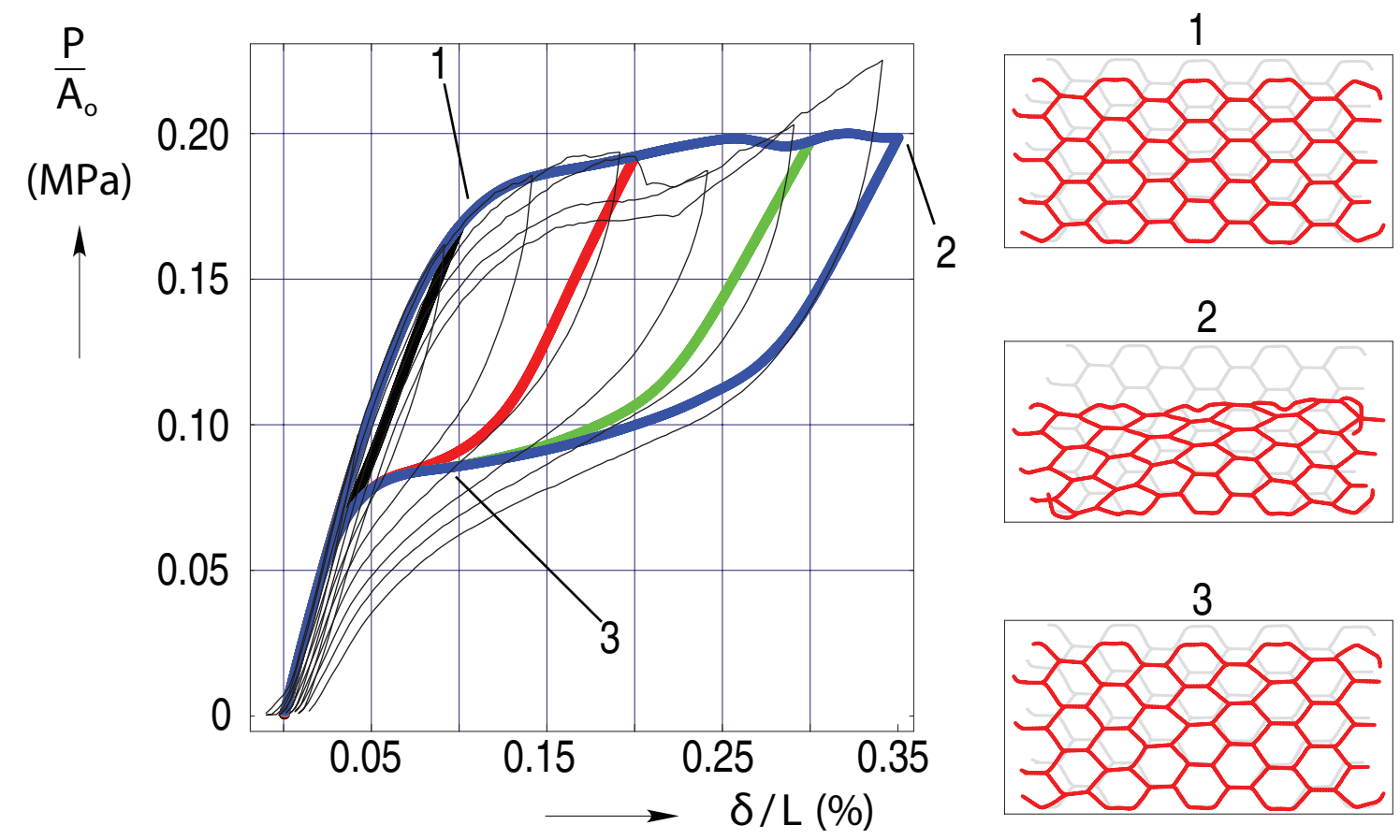

2
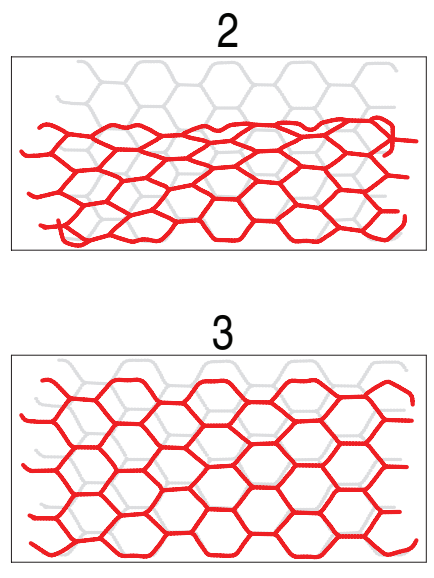

Figure 6. Results from numerical calculations: Response of finite element model for various loadings-unloadings to $10 \%, 20 \%, 30 \%$ and $35 \%$, superimposed on the data from the actual experiment, and on the right configurations of model for the specific points depicted in the response curve.

An incremental Newton-Raphson method is adopted for the numerical calculations reported here where each beam ligament is discretized in 10 elements in the $x$-direction with 4 d.o.f per node $\left(v, v_{, x}, w, w_{, x}\right)$ and Hermitian cubic interpolation for $v(x)$ and $w(x)$. A 4 point Gauss integration is used in each elements and at each integration station 51 equidistant $z$ points are used for the required thickness integrations. For each material point $(x, z)$ used in the calculations it's internal variable $\xi$ is also stored.

The accuracy of the code has been verified by analytical calculations on single ligaments and by comparing results in the loading regime of the honeycomb's response with previous calculations reported in Ref. 9.

The results of our calculations are shown in Fig. 6. Prior to their discussion and comparison with the experiments, two points should be made. The first concerns the selection of the ligament's constitutive response which is depicted in Fig. 5a. The numerical values for $E, E_{t}, \epsilon_{1 u}, \epsilon_{2 l}, \beta$ for the tensile and compressive regimes have been selected so as to provide a reasonable match between theory and experiment as depicted in Fig. 6. The second point is that the imperfect geometry of the specimen has been accurately represented in our calculations by taking into account the actual shape of each ligament.

Notice from the comparison between theory and experiment Fig. 6 that the macroscopic stress-strain response of the specimen is followed reasonably well for the loading phase. Moreover our simulation captures the transition of a relatively uniform strain (Fig. $6-1$ at $\delta / L=10 \%$ ) to a highly nonuniform deformation mode (Fig. 6-2 at $\delta / L=35 \%$ ). Observe that the large macroscopic strain mode is similar to the experimentally observed mode in Fig. 2b and captures the trend of strain distribution in vertical direction (although the experimental result depicted is for a larger macroscopic strain $\delta / L=52 \%)$. Upon unloading the more-or-less uniform deformation pattern is observed again in Fig. 6-3 which is similar to Fig. 6-1.

There is a rather important discrepancy between the numerically calculated and experimentally observed unloading paths, which can be explained by the presence of friction at the boundaries, a feature that is presently under investigation. On the overall the simulations show a reasonably good agreement with experiments.

\section{Conclusions}

It is shown for the first time that built-up honeycomb structures with $<5 \%$ relative density, fabricated from conventional wrought Nitinol material, exhibit an amplified form of superelasticity and shape memory 
when subjected to in-plane compressive loading. Long-standing difficulties with bonding Nitinol to itself were resolved with a newly developed brazing technique using niobium. We expect that a ten-fold improvement in thermal response rate can also be shown in the future due to the favorable scaling of material surface area/volume ratio. The findings so far have broad potential implications for lightweight armor, general energy absorption materials, compliance-matched biomedical implants, high-displacement actuators, and smart/adaptive structures. The design opportunities and thermo-mechanical behavior of light-weight cellular Nitinol structures are relatively unexplored, and there exist many interesting scientific questions and engineering challenges that will be pursued. Furthermore, now that Nitinol can be realized as open-cell, layered structures by simply building them up from off-the-shelf wrought SMA sheets, strips, tubes, or wires, such materials could be economically attractive.

\section{Acknowledgments}

J. Shaw and C. Churchill acknowledge the financial support of the Office of Naval Research (ONR young investigator grant, N00014-01-1-0581), the National Science Foundation (CAREER grant, CMS-9984189, grant CMS-0409172, and grant CMS-0409084), and the General Motors/University of Michigan Collaborative Research Laboratory for Smart Materials and Structures with sincere thanks. N. Triantafyllidis and P. Michailidis acknowledge support of National Science Foundation (grant CMS-0409084). D. Grummon and J. Foltz acknowledge the support of the Materials and Processes Laboratory at General Motors Research and Development.

\section{References}

\footnotetext{
${ }^{1}$ Gibson, L. J. and Ashby, M. F., Cellular Solids: Structure and Properties, Cambridge Solid State Series, Cambridge University Press, Cambridge, UK, 2nd ed., 1997.

${ }^{2}$ Ashby, M., Evans, A., Fleck, N., Gibson, L., Hutchinson, J., and Wadley, H., Metals Foams: A Design Guide, ButterworthHeinemann, Boston, MA, 1st ed., 2000.

${ }^{3}$ Lagoudas, D., Entchev, P., Vandygriff, E., Qidwai, M., and DeGiorgi, V., "Modeling of Thermomechanical Response of Porous Shape Memory Alloys," Proceedings of the Adaptive Structures and Material Systems Symposium, ASME International Mechanical Engineering Congress and Exposition, 2001.

${ }^{4}$ Shaw, J. A. and Kyriakides, S., "Thermomechanical Aspects of NiTi," Journal of the Mechanics and Physics of Solids, Vol. 43, No. 8, 1995, pp. 1243-1281.

${ }^{5}$ Shaw, J. A. and Kyriakides, S., "On the Nucleation and Propagation of Phase Transformation Fronts in a NiTi Alloy," Acta Materialia, Vol. 45, No. 2, 1997, pp. 683-700.

${ }^{6}$ Iadicola, M. A. and Shaw, J. A., "Rate and Thermal Sensitivities of Unstable Transformation Behavior in a Shape Memory Alloy," International Journal of Plasticity, Vol. 20, 2004, pp. 577-605.

${ }^{7}$ Papka, S. and Kyriakides, S., "In-plane Compressive Response of Crushing of Honeycomb," Journal of the Mechanics and Physics of Solids, Vol. 42, 1994, pp. 1499-532.

${ }^{8}$ Shaw, J. A., Grummon, D. S., and Foltz, J., "Superelastic NiTi Honeycombs: Fabrication and Experiments," Smart Materials and Structures, Vol. (to appear), 2006.

${ }^{9}$ Triantafyllidis, N. and Schraad, M., "Onset of Failure in Aluminum Honeycombs Under Arbitrary In-Plane Loading," Journal of the Mechanics and Physics of Solids, Vol. 46, 1998, pp. 1089-1124.
} 